\title{
ESTATÍSTICA NA AVALIAÇÃO DA QUALIDADE DA ÁGUA
}

\author{
Ana Luísa de Castro Pereira MARTINS \\ Paulo César de Resende ANDRADE² \\ José Izaquiel Santos da SILVA ${ }^{3}$
}

\author{
${ }^{1}$ Graduanda em Ciência e Tecnologia da Univ. Fed. dos Vales do Jequitinhonha e Mucuri, Diamantina - MG, Brasil \\ ${ }^{1,2}$ Professores da Univ. Fed. dos Vales do Jequitinhonha e Mucuri, Diamantina - MG, Brasil \\ E-mails para contato: analuisadecastro@yahoo.com.br; paulo.andrade@ict.ufvjm.edu.br; izaquiel@ict.ufvjm.edu.br
}

Recebido em: 0705/2015 - Aprovado em: 18/09/2015 - Disponibilizado em: 30/10/2015

\begin{abstract}
RESUMO
Este trabalho visa a implementação de uma modificação no teste de Tukey para comparações múltiplas, através da utilização de dados apresentados por Gonçalves (2009). No trabalho de Gonçalves foi realizada a avaliação da qualidade da água do rio Uberabinha, localizado em Uberlândia - MG. Gonçalves analisou diversos parâmetros de qualidade da água em cinco pontos localizados ao decorrer do rio, e assim, pode classificar o rio Uberabinha quanto a qualidade de sua água. Para as análises estatísticas Gonçalves empregou o teste de Tukey. Os mesmos dados foram utilizados neste trabalho para que se pudesse comparar as análises estatísticas feitas por Gonçalves com as análises estatísticas feitas pelo teste de tukey modificado, proposto por Andrade \& Ferreira (2010). Utilizou-se de dois parâmetros para fazer tal comparação. As análises estatísticas foram feitas através do programa R. Após comparar os resultados de Gonçalves com os resultados obtidos utilizando o teste de Tukey modificado, pode-se concluir que este é satisfatório.
\end{abstract}

PALAVRAS-CHAVE: Qualidade da água. Parâmetros de qualidade da água. Teste de Tukey. Análise estatística. Comparações múltiplas.

\section{ABSTRACT}

This work aims to implement a modification of Tukey test for multiple comparisons, using data presented by Gonçalves (2009). In Gonçalves' work was to performed the assessment of water quality of Uberabinha river. This river is located in Uberlândia - MG. Gonçalves analyzed several parameters of water quality in five points located all over the river, and thus he could classify the river Uberabinha according to the quality of its water. Gonçalves used the Tukey test to run statistical analysis. The same data were used in this study; therefore, it is possible to compare the statistical analysis made by Tukey test with statistical analyzes made by the modified Tukey test, proposed by Andrade \& Ferreira (2010). Two parameters were used to make this comparison. Statistical analyzes were made through the program R. After comparing the results of Gonçalves with the results obtained using the modified Tukey test, it can be concluded that this is satisfactory.

KEYWORDS: Water quality. Water quality parameters. Tukey test. Statistical analysis. Multiple comparisons.

\section{INTRODUÇÃO}

A água é um bem abundante no planeta Terra. Contudo os meios que esta é encontrada na natureza, 97\% água salgada, $2 \%$ de geleiras e $1 \%$ doce, faz com que a quantidade que pode ser consumida por humanos e animais seja de acesso restrito (ANA, 2003; Gonçalves, 2005). Essa pequena quantidade que está disponível para consumo nem sempre é aproveitada, devido à qualidade que esta água apresenta. 
De acordo com a RESOLUÇÃO CONAMA (Conselho Nacional do Meio Ambiente) $\mathrm{N}^{\circ}$ 20, de 18 de junho de 1986, a água é classificada em doces, salobras e salinas. As águas doces devem conter salinidade inferior ou igual a $0,5 \%$, as águas salobras $0,5 \%$ a $30 \%$ de salinidade, e as águas salinas devem ser igual ou superior a $30 \%$ de salinidade. A qualidade dessas águas leva em consideração o uso mais importante que essa água é destinada.

Para que se caracterize a qualidade da água, é necessária a obtenção de alguns parâmetros. A partir desses parâmetros podese calcular índices da qualidade da água para, assim, definir em qual categoria ela se encontra. Este Índice de Qualidade das Águas (IQA) foi criado em 1970, nos Estados Unidos, pela National Sanitation Foundation e começou a ser utilizado no Brasil em 1975, pela CETESB (Companhia Ambiental do Estado de São Paulo). Nos dias atuais, é o principal índice de qualidade utilizado no país. De acordo com a National Sanitation Foundation, o IQA depende de nove parâmetros, que possuem os seus respectivos $\operatorname{pesos}(w)$ que foram adotados em função da sua importância para a qualidade da água, como pode ser visualizado na Tabela 1

Tabela 1- Parâmetros de Qualidade da Água do IQA e respectivo peso.

\begin{tabular}{c|c}
\hline Parâmetro De Qualidade Da Água & Peso (W) \\
\hline Oxigênio dissolvido & 0,17 \\
Coliformes termotolerantes & 0,15 \\
Potencial hidrogeniônico - pH & 0,12 \\
Demanda Bioquímica de Oxigênio - DBO5,20 & 0,10 \\
Temperatura da água & 0,10 \\
Nitrogênio total & 0,10 \\
Fósforo total & 0,10 \\
Turbidez & 0,08 \\
Resíduo total & 0,08 \\
\hline
\end{tabular}

Fonte: Portal da Qualidade das Águas 
A descrição de cada parâmetro listado acima pode ser encontrada no site da Agência Nacional de Águas (ANA).

O IQA resume os dados paramétricos obtidos de uma determinada água, facilitando assim o entendimento sobre a qualidade desta, e possibilitando maiores informações em relação às possíveis necessidades de um tratamento por motivos de contaminação. De acordo com cada estado brasileiro, os valores do IQA são divididos em faixas, e estas são mostradas na Tabela 2.

Tabela 2- Faixas de classificação do IQA

\begin{tabular}{c|c|c}
\hline $\begin{array}{c}\text { Faixas de IQA utilizadas nos } \\
\text { seguintes Estados: AL, MG, } \\
\text { MT, PR, RJ, RN, RS }\end{array}$ & $\begin{array}{c}\text { Faixas de IQA utilizadas nos } \\
\text { seguinte Estados: BA, CE, ES, } \\
\text { GO, MS, PB, PE, SP }\end{array}$ & Avaliação da Qualidade da Água \\
\hline $91-100$ & $80-100$ & Ótima \\
$71-90$ & $52-79$ & Boa \\
$51-70$ & $37-51$ & Razoável \\
$26-50$ & $20-36$ & Ruim \\
$0-25$ & $0-19$ & Péssima \\
\hline
\end{tabular}

Fonte: Portal da Qualidade das Águas

Devido ao avanço da tecnologia e ao surgimento de indústrias em todo o mundo, a água está sendo utilizada abundantemente. Com este aumento do consumo, a água está perdendo sua qualidade devido ao uso inadequado. Com isso, há uma crescente preocupação com a qualidade da água, ocasionando numa necessidade de normas que busquem a preservação dos corpos hídricos.

Os padrões nacionais de qualidade a serem utilizados são definidos de acordo com cada país. As normas de qualidade da água no Brasil são descritas em resoluções apresentadas pelo Conselho Nacional do Meio
Ambiente (CONAMA). Estas resoluções possuem padrões tanto para os corpos receptores quanto para o lançamento de efluentes nos corpos d'agua. E ambos buscam garantir a preservação da qualidade da água. Estes padrões estão inter-relacionados, já que os padrões de lançamentos devem garantir também os padrões do corpo receptor. Para garantir esses padrões deverão ocorrer estudos que analisem os impactos ambientais.

Empregando alguns métodos estatísticos, pode-se fazer análises que comprovem os verdadeiros impactos a 
determinado corpo d'água, utilizando para isto, dados coletados no mesmo.

Será utilizada a análise de variância (ANOVA) para avaliar se há diferenças entre os tratamentos, por meio do teste F (Machado et al., 2005).

A rejeição da hipótese $H_{0}$ nos leva a buscar procedimentos para testar se existem diferenças reais entre os tratamentos, utilizando de procedimentos de comparações múltiplas (PCM) (Hochberg \& Tamhane, 1987; Machado et al., 2005; Ramalho,

\section{MATERIAL E MÉTODOS}

Utilizou-se o trabalho de Gonçalves (2009), que visa analisar a qualidade da água do Rio Uberabinha, que passa através da cidade de Uberlândia no estado de Minas Gerais, para que fosse possível a comparação de testes estatísticos. Os dados contidos no trabalho de Gonçalves foram fornecidos pelo Departamento de Águas e Esgotos de Uberlândia (DMAE). Esses dados foram
Ferreira \& Oliveira, 2000; Andrade \& Ferreira, 2010).

Este trabalho tem por objetivo avaliar a significância de alguns parâmetros importantes (Oxigênio Dissolvido e Potencial Hidrogeniônico) para verificar a qualidade da água, utilizando o teste modificado de Tukey, proposto por (Andrade \& Ferreira, 2010) e confrontar com os resultados de Gonçalves (2009), usando a linguagem de programação R (R. Development Core Team, 2015).

obtidos por meio de análises de parâmetros físicos, químicos e biológicos feitas em amostras de águas retiradas do Rio Uberabinha durante o período de 2007 e 2008.

Realizaram-se análises em 5 pontos diferentes ao decorrer do rio. Os pontos de coleta de amostras estão apresentados no mapa na Figura 1. 
Figura 1- Pontos de coleta de amostras do Rio Uberabinha

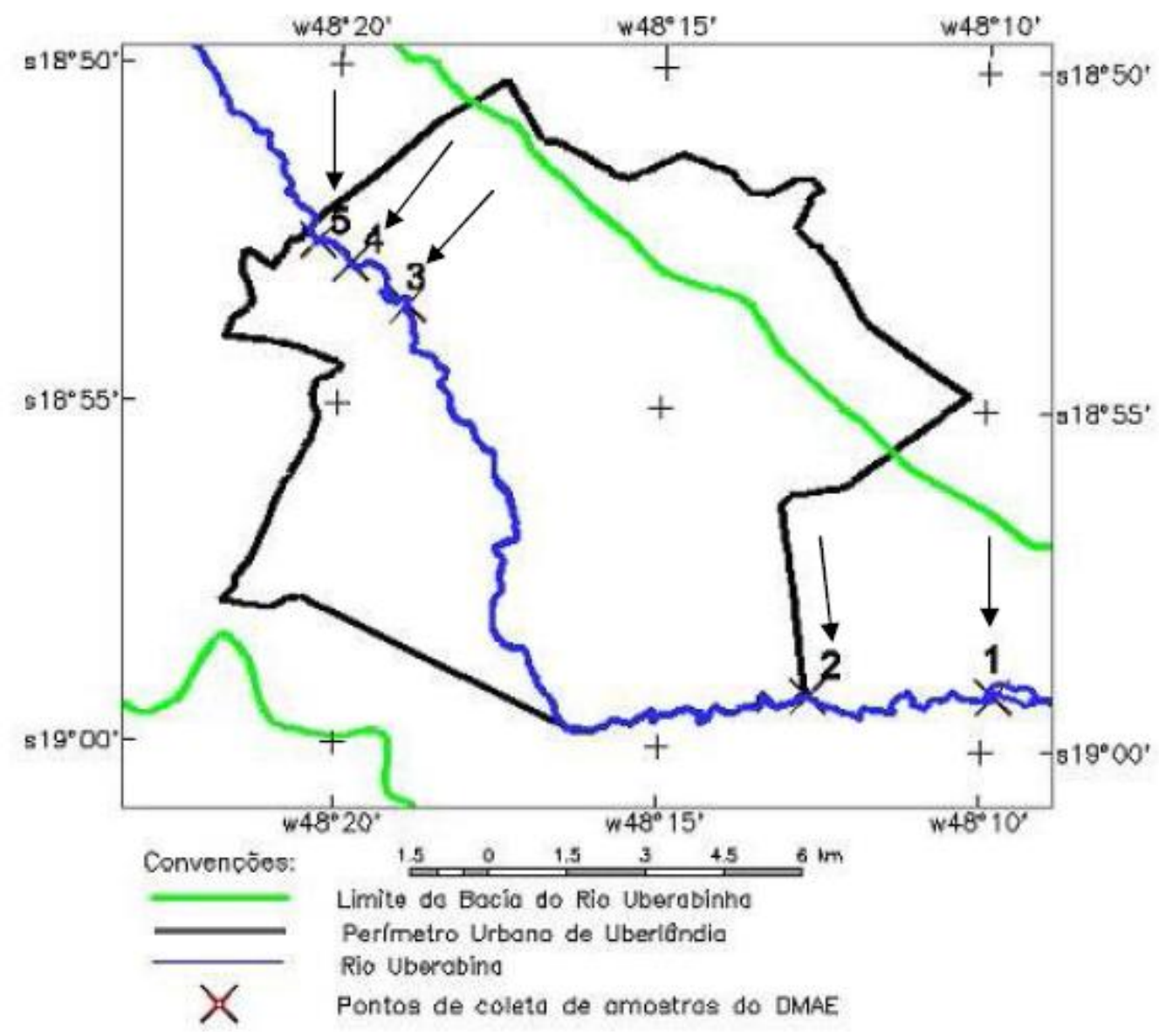

Fonte: Gonçalves (2009)

\section{RESULTADOS E DISCUSSÃO}

Realizando as análises de variância no programa $\mathrm{R}$, empregando a modificação do teste de Tukey, obtiveram-se alguns importantes resultados sobre os parâmetros oxigênio dissovido e potencial hidrogeniônico. Estes estão descritos a seguir.

\section{Oxigênio Dissolvido:}

Estão apresentados na Tabela 3 os dados sobre oxigênio dissolvido, contidos no trabalho de Gonçalves (2009).

A análise de variância considerando todos os meses dos anos de 2007 e 2008 para o oxigênio dissolvido está apresentada na Tabela 4. 
Tabela 3 - Oxigênio Dissolvido (mg/L), durante o monitoramento realizado pelo DMAE.

\begin{tabular}{cccccc}
\hline & Ponto 1 & Ponto 2 & Ponto 3 & Ponto 4 & Ponto 5 \\
\hline Fev/07 & 6,80 & 5,10 & 5,20 & 5,60 & 5,40 \\
Mai/07 & 6,80 & 7,20 & 6,50 & 6,40 & 6,00 \\
Ago/07 & 7,50 & 8,50 & 7,90 & 7,50 & 7,50 \\
Nov/07 & 7,30 & 7,40 & 5,20 & 6,50 & 6,00 \\
Fev/08 & 5,80 & 6,10 & 6,30 & 6,60 & 6,10 \\
Mai/08 & 5,30 & 6,20 & 6,80 & 6,90 & 6,20 \\
Ago/08 & 6,80 & 8,00 & 6,30 & 6,50 & 5,40 \\
Nov/08 & 5,80 & 7,60 & 7,70 & 8,00 & 7,50 \\
\hline
\end{tabular}

Tabela 4 - Análise de variância geral para OD

\begin{tabular}{llllll}
\hline & GL & SQ & QM & F & Pr \\
\hline Trat & 7 & 15.82 & 2.2604 & 4.698 & 0.00103 \\
Resíduos & 32 & 15.40 & 0.4811 & & \\
\hline Total & 39 & 31.22 & 00 & & \\
\hline
\end{tabular}

Através dos resultados acima, observase uma diferença significativa entre os meses, pois a probabilidade foi menor que $5 \%(\operatorname{Pr}$ $=0.00103)$. Esse resultado difere um dos apresentados por Gonçalves (2009). Assim, para descobrir onde está esta diferença, utilizou-se o teste de comparações múltiplas modificado de Tukey, via software R. O resultado pode ser observado na Figura 2. 
$95 \%$ family-wise confidence level

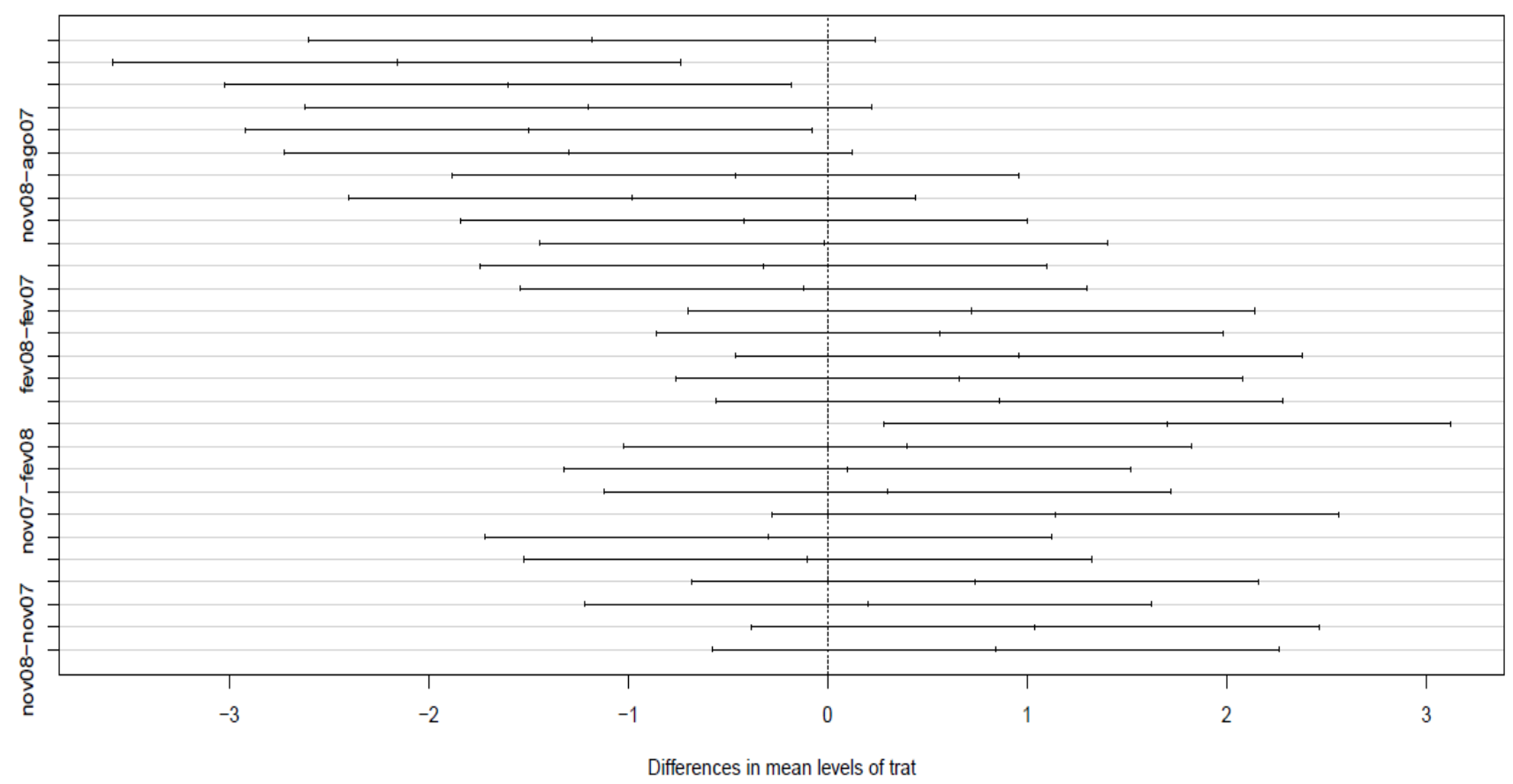

Fonte: Programa R

Observando a figura acima, conclui-se que houve diferença entre os meses de fevereiro e agosto de 2007, fevereiro de 2008 e agosto de 2007, maio de 2008 e agosto de 2007, novembro de 2008 e fevereiro de 07.

Tabela 5 - Análise de variância para OD em 2007
Foi feita uma análise também por ano. Para o ano de 2007, a análise de variância está representada da Tabela 5.

\begin{tabular}{llllll}
\hline & GL & SQ & QM & F & Pr \\
\hline Trat & 3 & 11.834 & 3.945 & 9.211 & 0.000896 \\
Resíduos & 16 & 6.852 & 0.428 & & \\
\hline Total & 19 & 18.686 & & & \\
\hline
\end{tabular}


Como a probabilidade encontrada foi menor que 5\%, conclui-se que há pelo menos uma diferença significativa entre os meses do ano de 2007. Para encontrá-la realizou o teste modificado de Tukey, no programa R, considerando $5 \%$ de significância, cujos resultados estão representados na Tabela 6 .

Tabela 6 - Intervalos de confiança a 95\%

\begin{tabular}{cccc}
\hline & lwr & upr & p adj \\
\hline fev07-ago07 & -3.344 & - & 0.0004 \\
& & 0.975 & \\
mai07-ago07 & -2.384 & - & 0.0464 \\
& & 0.015 & \\
nov07-ago07 & -2.484 & - & 0.0289 \\
& & 0.115 & \\
mai07-fev07 & -0.224 & 2.144 & 0.1349 \\
nov07-fev07 & -0.324 & 2.044 & 0.2022 \\
nov07-mai07 & -1.284 & 1.084 & 0.9948 \\
\hline
\end{tabular}

Observa-se que houve diferença entre o mês de agosto de 07 e os demais meses (fevereiro, maio e novembro de 07) pois o intervalo de confiança não contém o valor zero e a probabilidade foi menor que $5 \%$.

Para o ano de 2008, a análise de variância está representada da Tabela 7.

Tabela 7 - Análise de variância para OD em 2008

\begin{tabular}{llllll}
\hline & GL & SQ & QM & F & Pr \\
\hline Trat & 3 & 3.986 & 1.329 & 2.488 & 0.0976 \\
Resíduos & 16 & 8.544 & 0.534 & & \\
\hline Total & 19 & 12.530 & & & \\
\hline
\end{tabular}

Como a probabilidade encontrada é igual a 0.0976 , ou seja, maior que 5\%, não há diferença significativa entre os meses dentro do ano de 2008. Foi feita também uma análise comparando as médias de oxigênio dissolvido entre os anos de 2007 e 2008, por meio do 
teste $\mathrm{t}$ de comparação de médias e não foi constatada diferença significativa.

Além disso, fez-se uma comparação de cada mês dentro dos anos de 2007 e 2008, utilizando-se também o teste $\mathrm{t}$ de comparação de duas médias. Foi verificado que houve diferença significativa somente para o mês de agosto, conforme Tabela 8.

Tabela 8 - Análises mensais para OD, nos anos de 2007 e 2008.

\begin{tabular}{ccc}
\hline & Pr & Significância \\
\hline Fevereiro & 0.9337 & $\mathrm{~ns}$ \\
Maio & 0.2075 & $\mathrm{~ns}$ \\
Agosto & 0.01729 & $5 \%$ \\
Novembro & 0.9118 & $\mathrm{~ns}$ \\
\hline
\end{tabular}

ns significa não significativo

\section{Potencial Hidrogeniônico:}

Os dados do potencial hidrogeniônico

(pH) (Gonçalves, 2009), estão na Tabela 9.

Tabela 9 - Potencial Hidrogeniônico durante o período de monitoramento realizado pelo DMAE.

\begin{tabular}{cccccc}
\hline & Ponto & Ponto & Ponto & Ponto & Ponto \\
& 1 & 2 & 3 & 4 & 5 \\
\hline Fev/07 & 6,31 & 6,50 & 6,50 & 6,60 & 6,60 \\
Mai/07 & 6,80 & 6,40 & 6,60 & 6,60 & 6,70 \\
Ago/07 & 6,87 & 7,50 & 7,30 & 7,40 & 7,30 \\
Nov/07 & 6,20 & 6,10 & 6,20 & 6,40 & 6,20 \\
Fev/08 & 6,07 & 6,40 & 6,70 & 6,80 & 6,70 \\
Mai/08 & 6,48 & 7,20 & 6,90 & 7,00 & 7,10 \\
Ago/08 & 6,85 & 7,30 & 6,60 & 6,50 & 5,40 \\
Nov/08 & 6,47 & 6,20 & 6,30 & 6,20 & 6,80
\end{tabular}

Fonte: Gonçalves (2009) 
A análise de variância considerando todos os meses dos anos de 2007 e 2008 para o potencial hidrogeniônico está apresentada na Tabela 10.

Tabela 10 - Análise de variância geral para pH

\begin{tabular}{llllll}
\hline & GL & SQ & QM & F & Pr \\
\hline Trat & 7 & 3.839 & 0.5484 & 5.277 & 0.000442 \\
Resíduos & 32 & 3.325 & 0.1039 & & \\
\hline Total & 39 & 7.164 & & & \\
\hline
\end{tabular}

Há diferença significativa entre os meses, pois a probabilidade foi menor que $5 \%$, Para encontrar onde está a diferença, usaFigura 3: Comparação entre as médias se $o$ teste de comparações múltiplas modificado de Tukey, cujos resultados estão apresentados na Figura 3.

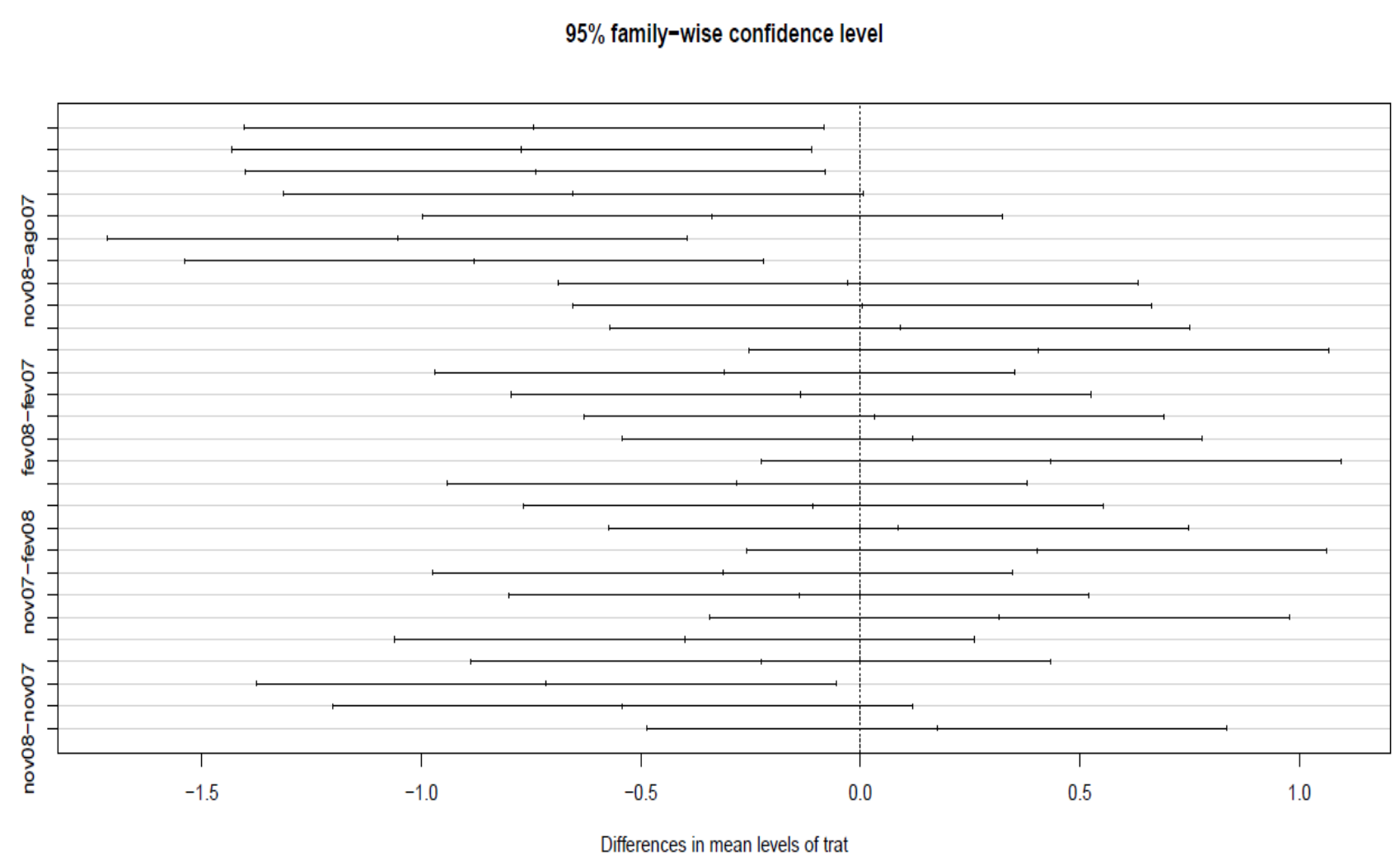

Fonte: Programa R 
Observa-se que houve diferença entre os meses de agosto de 2008 e agosto de 2007, fevereiro de 2007 e agosto de 2007, fevereiro de 2008 e agosto de 2007, novembro de 2007 e agosto de 2007, novembro de 2008 e agosto de 2007, novembro de 2007 e maio de 2008.

Fazendo uma análise por ano, verificase que para o ano de 2007, a análise de variância está representada da Tabela 11.

Tabela 11 - Análise de variância para pH em 2007

\begin{tabular}{llllll}
\hline & GL & SQ & QM & F & Pr \\
\hline Trat & 3 & 2.9851 & 0.9950 & 37.58 & $1.79 \mathrm{e}-07$ \\
Resíduos & 16 & 0.4236 & 0.0265 & & \\
\hline Total & 19 & 3.4087 & & & \\
\hline
\end{tabular}

Sendo a probabilidade menor que 5\%, há pelo menos uma diferença significativa entre os meses dentro do ano de 2007. Para saber onde está a diferença, aplica-se o teste modificado de Tukey, no programa R, considerando $5 \%$ de significância, conforme Tabela 12.

Tabela 12 - Intervalos de confiança a 95\%

\begin{tabular}{cccc}
\hline & lwr & upr & p adj \\
\hline fev07-ago07 & -1.066 & -0.477 & 0.0000 \\
mai07-ago07 & -0.948 & -0.359 & 0.0001 \\
nov07-ago07 & -1.348 & -0.759 & 0.0000 \\
mai07-fev07 & -0.176 & 0.412 & 0.6672 \\
nov07-fev07 & -0.576 & 0.012 & 0.0628 \\
nov07-mai07 & -0.694 & -0.105 & 0.0064 \\
\hline
\end{tabular}

Pode-se observar que há diferença está entre os meses de agosto de 07 com os demais (fevereiro, maio e novembro de 2007) e novembro de 07 e maio de 07.
Para o ano de 2008, a análise de variância está representada da Tabela 13 
Tabela 13 - Análise de variância para pH em 2008

\begin{tabular}{llllll}
\hline & GL & SQ & QM & F & Pr \\
\hline Trat & 3 & 0.8229 & 0.2743 & 1.512 & 0.249 \\
Resíduos & 16 & 2.9018 & 0.1814 & & \\
\hline Total & 19 & 3.7247 & & & \\
\hline
\end{tabular}

Verifica-se que não há diferença significativa entre os meses dentro do ano de 2008, pois o valor a probabilidade é maior que $5 \%$, ou seja, 0.249 .

Foi feita também uma análise comparando as médias de potencial hidrogeniônico, entre os anos de 2007 e 2008, por meio do teste $t$ de comparação de médias e não foi constatada diferença significativa.
Foi feita também uma comparação de cada mês dentro dos anos de 2007 e 2008, utilizando-se também o teste $\mathrm{t}$ de comparação de duas médias. Foi verificado que houve diferença significativa somente para o mês de agosto, conforme Tabela 14 .

Tabela 14 - Análises mensais para pH, nos anos de 2007 e 2008.

\begin{tabular}{ccc}
\hline & Pr & Significância \\
\hline Fevereiro & 0.5852 & $\mathrm{~ns}$ \\
Maio & 0.9723 & $\mathrm{~ns}$ \\
Agosto & 0.02778 & $5 \%$ \\
Novembro & 0.9025 & $\mathrm{~ns}$ \\
\hline
\end{tabular}

ns significa não significativo

\section{CONCLUSÃO}

Através das análises feitas utilizando dados de dois parâmetros, oxigênio dissolvido e potencial hidrogeniônico, apresentados por Gonçalves (2009), pode-se concluir que a modificação do teste de Tukey, proposta por (Andrade \& Ferreira, 2010) foi satisfatória.

Os resultados são similares aos apresentados por Gonçalves (2009) para o oxigênio dissolvido e potencial hidrogeniônico, apesar de apresentar algumas 
diferenças pontuais, que mostram uma maior capacidade de detecção de diferenças significativas do teste de Tukey modificado.

Outro ponto positivo é a facilidade de interpretação dos resultados, devido à diminuição da ambiguidade. Em todos os casos analisados a alternativa apresentada por Andrade \& Ferreira (2010) apresentou melhores resultados em relação ao teste de Tukey convencional.

O poder foi maior, ou seja, o teste modificado é mais eficiente na identificação das diferenças entre as médias dos tratamentos. Sendo assim, realmente apresenta resultados satisfatórios quando comparado com o teste de Tukey.

\section{REFERÊNCIAS}

1. ANA. Agência Nacional de Águas. Águas Subterrâneas. Disponível em <www.ana.gov.br/.../aguasSubterr/Est udoAguasSubterraneasANA22-0802.doc> Acessado em: 15 de maio de 2009.

2. ANDRADE, P. C. R.; FERREIRA, D. F. Comparações múltiplas bayesianas em modelos normais homocedásticos $\mathrm{e}$ heterocedásticos. Ciência e
Agrotecnologia, Lavras, v.34, n.4, p.845852, jul./ago., 2010.

3. CONAMA. Conselho Nacional do Meio Ambiente.

<http://www.mma.gov.br/port/conama /principal.cfm> Acessado em: $15 \mathrm{de}$ abril de 2015.

4. GONÇALVES, E M. Avaliação da Qualidade da Água do Rio Uberabinha - Uberlândia-MG. 2009. 141 f. Dissertação (Mestrado em Tecnologia dos Processos Químicos) Escola de Química, Universidade Federal do Rio de Janeiro, Rio de Janeiro. 2009.

5. HOCHBERG, Y.; TAMHANE, A.C. Multiple comparisons procedures. New York: John Wiley, 1987. 450p.

6. MACHADO, A.A.; DEMÉTRIO, C.G.B.; FERREIRA, D.F.; SILVA, J.G.C. Estatística experimental: uma abordagem fundamentada no planejamento e no uso de recursos computacionais. In: REUNIÃO ANUAL DA REGIÃO BRASILEIRA DA SOCIEDADE INTERNACIONAL DE BIOMETRIA, 50; SIMPÓSIO DE ESTATÍSTICA APLICADA À 


\section{EXPERIMENTAÇÃO}

AGRONÔMICA, 11., Londrina, 2005.

Curso... Londrina: ISBN, 2005. 290p.

7. Portal da Qualidade das Aguas.

Disponível em:

$<$ http://pnqa.ana.gov.br/indicadores-

indice-aguas.aspx > Acessado em: 15

de abril de 2015 .

8. RAMALHO, M.A.P.; FERREIRA, D.F.; OLIVEIRA, A.C.

Experimentação em genética e melhoramento de plantas. Lavras:

UFLA, 2000. 303p.
9. R Development Core Team. R: A language and environment for statistical computing. Vienna, Austria: R Foundation for Statistical Computing, 2015.

10. SPERLING, Marcos. Introdução à Qualidade das Águas e ao Tratamento de Esgotos (Princípios do Tratamento Biológico de Águas Residuárias; vol. 1). Belo Horizonte: DESA-UFMG, 452p. 2005. 